\title{
Letrozole-Induced Very Early Systemic Sclerosis in a Patient With Breast Cancer: A Case Report
}

\author{
Gabriel POKHAI, Rino BUZZOLA, Adriana ABRUDESCU \\ Department of Internal Medicine, Mount Sinai School of Medicine, Queens Hospital Center, Jamaica, NY, USA
}

\begin{abstract}
Aromatase inhibitors (Als) are standard therapy for post-menopausal women with breast cancer. The use of Als has been strongly associated with musculoskeletal symptoms and with various autoimmune conditions recently. Systemic sclerosis (SSc) is a connective tissue disorder of unknown etiology which may present with a broad range of clinical manifestations. In SSc, matrix deposition by activated fibroblasts leads to vasculopathy, systemic fibrosis, and end-organ damage. Skin, kidneys, pulmonary and gastrointestinal systems may be affected. Very early SSc can be diagnosed in patients with Raynaud's phenomenon and puffy fingers as well as through autoantibodies and nailfold capillaroscopy. These symptoms are highly predictive of the further development of definite SSc. In this article, we report an unusual case of a 61-year-old female breast cancer with a very early-onset SSc with bilateral stiffness of the hands, Raynaud's phenomenon, puffy fingers, dilated nailfold capillaries and positive anticentromere-B antibody titers after undergoing $\mathrm{Al}$ therapy with letrozole. Substitution of letrozole with exemestane followed by eventual discontinuation of Al therapy appears to relieve symptoms. To the best of our knowledge, this is the first report suggesting a link between Al use and SSc. We suggest that Al use may induce SSc, or at least, exacerbate existing very early SSc in post-menopausal patients with breast cancer.

Keywords: Aromatase inhibitor; breast cancer; letrozole; scleroderma; very early systemic sclerosis.
\end{abstract}

Aromatase inhibitors (AIs) are currently the standard of treatment in postmenopausal women with estrogen-receptor-positive breast cancer. The AIs inhibit the conversion of androstenedione into estrogens, thus suppressing circulating estradiol levels. ${ }^{1}$ There are three available AIs: anastrozole, letrozole, and exemestane.

Systemic sclerosis (SSc) is a connective tissue disorder of unknown etiology that can have widespread and varied clinical manifestations. In $\mathrm{SSc}$, matrix deposition by activated fibroblasts leads to vasculopathy, systemic fibrosis, and endorgan damage. ${ }^{2}$ Survival is $55 \%$ at 10 years and is negatively impacted by the extent of skin, renal, pulmonary and gastrointestinal organ system involvement. ${ }^{3}$ A very early diagnosis of SSc (very early SSc) can be made in patients with Raynaud's phenomenon (RP), puffy fingers, autoantibodies, and characteristic nailfold capillaroscopy. ${ }^{4}$

In this article, we describe a female patient with a history of breast cancer and a possible case of letrozole-induced very early SSc.

\section{CASE REPORT}

A 61-year-old female patient with a medical history of hypertension and breast cancer was referred to our hospital in August 2011. She underwent mastectomy in 2007, and had since been on AI therapy with letrozole. She presented with a history of worsening joint pain and stiffness in both hands since 2009. The patient was unable to perform daily activities such as turning

\footnotetext{
Received: February 22, 2013 Accepted: May 26, 2013

Correspondence: Gabriel Pokhai, M.D. Department of Internal Medicine, Mount Sinai School of Medicine, Queens Hospital Center, 11432 Jamaica, NY, USA. Tel: +1 7188833000 e-mail: gpokhai@hotmail.com
} 
a door knob or actions like making a fist. The patient was previously seen by a rheumatologist who prescribed hydroxychloroquine for synovitis without any improvement of her symptoms in a private healthcare center. Since 2010, the patient experienced bilateral indurations of the dorsum of the hands and associated vasospastic phenomena exacerbated by exposure to low temperature with her distal phalanges undergoing discoloration from white to blue to red consistent with RP.

Physical examination was remarkable for puffy, swollen digits (Figure 1) and nail fold changes consisting of dilated capillary loops at the base of the nails bilaterally of all fingers, which were clearly visible to the naked eye (Figure 2). The proximal interphalangeal joints were tender bilaterally with palpable synovitis.

Based on rheumatologic and seroimmunological examination findings, the patient was found to have a high titer of antinuclear antibody (1:1280) with a centromeric pattern, and positive anticentromere- $\mathrm{B}$ antibodies (>8.0 AI). The following antibodies were negative: doublestranded DNA, anti-Smith, ribonucleoprotein, Ro-La, and rheumatoid factor. Normal levels of C3, C4 and C-reactive protein were present and erythrocyte sedimentation rate was not elevated.

Due to worsening RP and joint pain, letrozole was discontinued and the patient was switched to another AI (exemestane). During the follow-up visit a few months following letrozole discontinuation, the patient reported improvement in joint pain with increased functional capacity. Steroidal AI therapy was eventually discontinued completely in 2012. Subsequently, she was able to perform

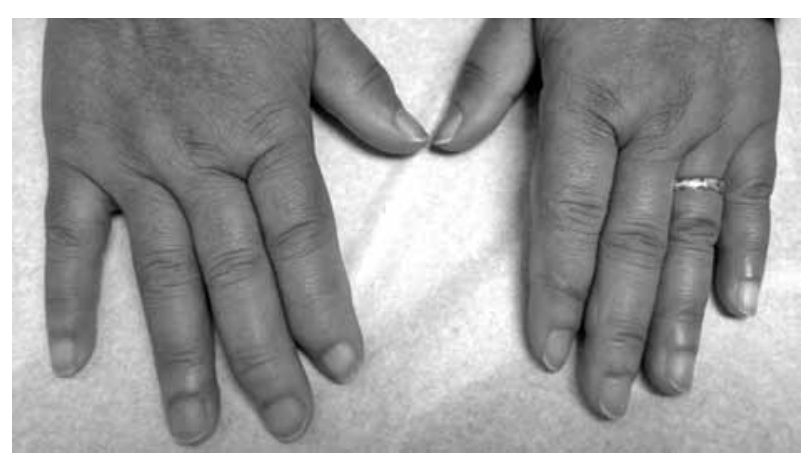

Figure 1. The patient's hands, displaying puffy, swollen digits. daily activities and experienced less vasospastic changes of the fingers on cold exposure. Physical examination showed less induration of the skin, resolution of the periungual erythema, and increased range of movement of the fingers.

\section{DISCUSSION}

Aromatase inhibitors are used in the treatment of post-menopausal women with receptor-positive breast malignancies in both metastatic and adjuvant settings. The most common adverse effects associated with $\mathrm{AI}$ are musculoskeletal symptoms including joint pain and stiffness. ${ }^{5}$ There have also been cases of rheumatological and autoimmune conditions described in the literature, which have been attributed to the $\mathrm{AI}$ use. These include cutaneous vasculitis, ${ }^{6}$ subacute cutaneous lupus erythematosus, ${ }^{7}$ rheumatoid arthritis, ${ }^{8}$ Sjögren-like syndromes, ${ }^{9}$ and immune-mediated hepatitis. ${ }^{10}$ To the best of our knowledge, there are no cases reported in the literature of $\mathrm{SSc}$ in conjunction with $\mathrm{AI}$ use.

Systemic sclerosis is a non-inflammatory connective tissue disorder with diffuse small vessel fibrosis and vasculopathy, as well as fibrosis of the skin and internal organs. Clinical manifestations are wide-ranging and may include dermatological, gastrointestinal, renal, pulmonary, vascular, and musculoskeletal systems. Systemic sclerosis is divided into two subsets of disease based on the extent of skin tightening. Limited cutaneous SSc, formerly called CREST syndrome, is a subset of SSc featuring RP, and thickening of the skin confined to the face, neck and extremities without

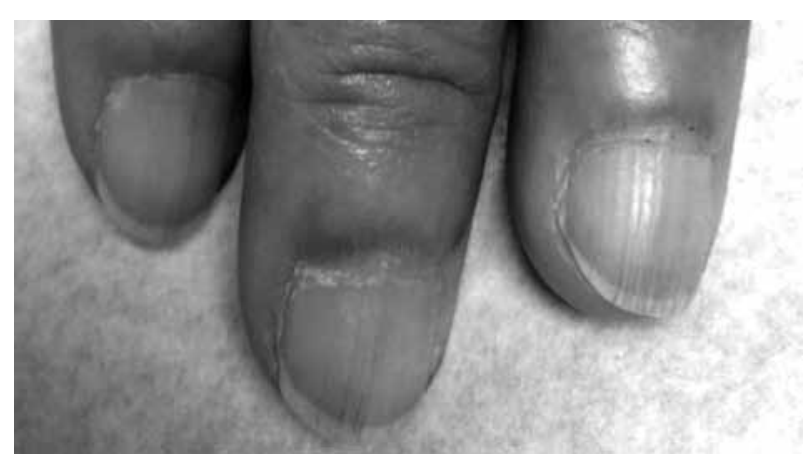

Figure 2. A close-up of the patient's nailbeds, showing dilated capillary loops. 
visceral involvement. In diffuse cutaneous SSc, the skin of the proximal extremities and trunk is also involved. ${ }^{11}$

Although the etiology remains unknown, there are well-documented risk factors such as female sex, human leukocyte antigen (HLA)-DR1 haplotype, and exposure to silica and aromatic hydrocarbons. Several medications have been known to induce scleroderma-like clinical conditions including bleomycin, docetaxel, and penicillamine. ${ }^{12}$ In addition, one case report described SSc possibly induced by interferon alpha and interleukin 2 (IL-2) treatment in a patient with metastatic melanoma. ${ }^{13}$

To aid clinicians in the diagnosis of SSc before end-organ damage occurs, the European League Against Rheumatism has released preliminary criteria for the diagnosis of very early SSc. The list includes RP, puffy swollen digits, abnormal capillaroscopy with scleroderma pattern, and positive antibodies (anticentromere and antitopoisomerase-1). ${ }^{4}$ Another study showed that in patients with the aforementioned signs and symptoms, there was a probability of $79.5 \%$ of developing definite SSc after nine years of observation. ${ }^{14}$

In this case, the patient described discoloration of the digits fitting the classic pattern of RP. The history of $\mathrm{RP}$ and the clinical findings of puffy fingers, dilated nailfold capillaries, and positive anti-centromere titers meet the EULAR criteria for very early SSc.

It is possible that the patient could have had pre-existing very early SSc and remained asymptomatic until the time of AI therapy. Another possibility is that the very early SSc was induced by paraneoplastic causes. An extensive meta-analysis of the literature by Bonifazi et al. ${ }^{15}$ suggested an increased frequency of cancers, lung and hematological neoplasms particularly, in patients with SSc. However, a clear and statistically significant association with breast cancer could not be confirmed, and studies failed to establish a causal relationship between cancer and SSc. ${ }^{16}$

Given the fact that these findings occurred after starting letrozole, along with their subsequent abatement after discontinuing it, we may suggest a possible association between SSc and the use of letrozole. Applying the Naranjo adverse drug reaction probability scale ${ }^{17}$ to this case, a score of 6 was obtained, indicating a probable adverse drug reaction. This case suggests that AI use may induce, or at the very least, exacerbate very early SSc in patients.

\section{Declaration of conflicting interests}

The authors declared no conflicts of interest with respect to the authorship and/or publication of this article.

\section{Funding}

The authors received no financial support for the research and/or authorship of this article.

\section{REFERENCES}

1. Adelson K, Germain D, Raptis G, Biran N. Hormonal modulation in the treatment of breast cancer. Endocrinol Metab Clin North Am 2011;40:519-32.

2. Varga J, Abraham D. Systemic sclerosis: a prototypic multisystem fibrotic disorder. J Clin Invest 2007;117:557-67.

3. Mayes MD, Lacey JV Jr, Beebe-Dimmer J, Gillespie BW, Cooper B, Laing TJ, et al. Prevalence, incidence, survival, and disease characteristics of systemic sclerosis in a large US population. Arthritis Rheum 2003;48:2246-55.

4. Avouac J, Fransen J, Walker UA, Riccieri V, Smith $\mathrm{V}$, Muller $\mathrm{C}$, et al. Preliminary criteria for the very early diagnosis of systemic sclerosis: results of a Delphi Consensus Study from EULAR Scleroderma Trials and Research Group. Ann Rheum Dis 2011;70:476-81.

5. Salgado BA, Zivian MT. Aromatase inhibitors: side effects reported by 622 women. Breast Cancer Res Treat 2006;100:S168.

6. Santoro S, Santini M, Pepe C, Tognetti E, Cortelazzi C, Ficarelli E, et al. Aromatase inhibitor-induced skin adverse reactions: exemestane-related cutaneous vasculitis. J Eur Acad Dermatol Venereol 2011;25:596-8.

7. Trancart M, Cavailhes A, Balme B, Skowron F. Anastrozole-induced subacute cutaneous lupus erythematosus. Br J Dermatol 2008;158:628-9.

8. Bertolini E, Letho-Gyselinck H, Prati C, Wendling D. Rheumatoid arthritis and aromatase inhibitors. Joint Bone Spine 2011;78:62-4.

9. Laroche M, Borg S, Lassoued S, De Lafontan B, Roché $\mathrm{H}$. Joint pain with aromatase inhibitors: abnormal frequency of Sjögren's syndrome. J Rheumatol 2007;34:2259-63.

10. Inno A, Basso M, Vecchio FM, Marsico VA, Cerchiaro E, D'Argento E, et al. Anastrozole-related acute hepatitis with autoimmune features: a case report. BMC Gastroenterol 2011;11:32. 
11. LeRoy EC, Black C, Fleischmajer R, Jablonska S, Krieg T, Medsger TA Jr, et al. Scleroderma (systemic sclerosis): classification, subsets and pathogenesis. J Rheumatol 1988;15:202-5.

12. Haustein UF, Haupt B. Drug-induced scleroderma and sclerodermiform conditions. Clin Dermatol 1998;16:353-66.

13. Böni R, Dummer R, Burg G. Acral sclerosis and leukoderma in a melanoma patient treated with interferon alpha and interleukin-2. Eur $\mathrm{J}$ Dermatol 1995;5:383-5.

14. Koenig M, Joyal F, Fritzler MJ, Roussin A, Abrahamowicz M, Boire G, et al. Autoantibodies and microvascular damage are independent predictive factors for the progression of Raynaud's phenomenon to systemic sclerosis: a twenty-year prospective study of 586 patients, with validation of proposed criteria for early systemic sclerosis. Arthritis Rheum 2008;58:3902-12.

15. Bonifazi M, Tramacere I, Pomponio G, Gabrielli B, Avvedimento EV, La Vecchia C, et al. Systemic sclerosis (scleroderma) and cancer risk: systematic review and meta-analysis of observational studies. Rheumatology (Oxford) 2013;52:143-54.

16. Marasini B, Conciato L, Belloli L, Massarotti M. Systemic sclerosis and cancer. Int $\mathrm{J}$ Immunopathol Pharmacol 2009;22:573-8.

17. Naranjo CA, Busto U, Sellers EM, Sandor P, Ruiz I, Roberts EA, et al. A method for estimating the probability of adverse drug reactions. Clin Pharmacol Ther 1981;30:239-45. 